\title{
Research on FMCW Radar Signals Processing
}

\author{
LI Bin \\ Department of Information Engineering \\ Yangzhou Polytechnic College \\ Yangzhou, China \\ E-mail: yzjylb@yeah.net
}

\author{
YU Xiaodan \\ Logging Company of SINOPEC \\ East China Petroleum Bureau \\ Yangzhou, China
}

\begin{abstract}
Frequency Modulated Continuous Wave radar or FMCW radar has obvious advantages on its low emitting power, strong resistance to inference signals, compact in size and high precision, therefore it is becoming widely used for distance and velocity measurement. The resolution of the FMCW radar is largely determined by the linearity of the Linear FrequencyModulated (LFM) signal generated by the VCO, even if minor nonlinearity may lead to severe deterioration to the resolution of the system, accordingly the linearity of LFM signal is vital to the system overall performance. In real practice, however, the Voltage-Frequency curve of VCO more or less suffered from nonlinearity, so we must compensate its nonlinearity before a FMCW radar can be used.This paper compensate the nonlinearity of VCO operates on $60 \mathrm{GHz}$ wave band. We have designed a practical method, and implemented hardware experiments.
\end{abstract}

Keywords- FMCW radar;Millimeter wave radar;VCO nonlinearity compensation;FPGA

\section{INTRODUCTION}

\section{A. Brief Description of FMCW Radar}

FMCW Radar has the advantages of low emission power, strong anti-jamming, small, light, high precision. So it's widely applied in such fields as precision guidance, automobile collision warning, navigation and traffic control, target searching and acquisition ${ }^{[1][2]}$. In one cycle, the Radar transmits a continuous wave in a constant direction and the frequency of wave increases linearly as time went and receives echoes continuously from the direction through another antenna ${ }^{[3]}$.

The frequency of FMCW Radar mentioned in this paper is about $60 \mathrm{GHz}$. $60 \mathrm{GHz}$-band is located in atmospheric absorption window. In bandwidth range of $8 \mathrm{GHz}$ near $60 \mathrm{GHz}$, atmospheric attenuation is $10-15 \mathrm{~dB} / \mathrm{km}$ which is fit for short distance $(<1 \mathrm{~km})$, high density, large capacity wireless communication ,especially for wireless sensor network, automobile collision warning radar and so on and remain a major concern these years ${ }^{[4]}$. The FCC has applied $57-62 \mathrm{GHz}$ band in industry, science and medical treatment. Japan and European countries will also open $59-62 \mathrm{GHz}$ band at least ${ }^{[5]}$.

\section{B. Ranging principle of FMCW Radar}

The schematic diagram of typical FMCW radar is as Figure 1 shown. Its emission signal is a linear FM signal and its FM cycle is larger than the maximum echo delay. Generally, it's triangular signal and its frequency increase linearly with time in a cycle. The signal level and frequency characteristic are as shown in Figure 2. The millimeter wave signal produce delay and form echo $f_{t}\left(t-t_{d}\right)$ after reflection of target, and then received by another independent antenna through transmitter and mix with local-oscillator signal $f_{t}$, finally obtain IF signal $f_{b[6]}$

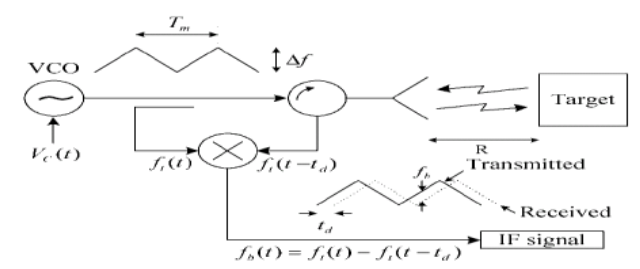

Figure 1. Schematic diagram of FMCW radar
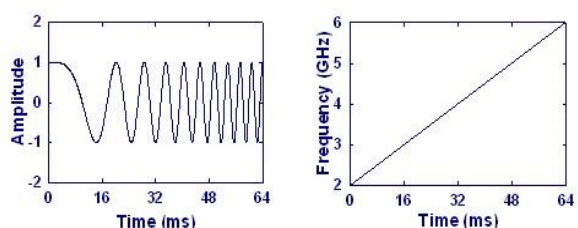

Figure 2. Relations between magnitude, frequency of FMCW radar and time

If the bandwidth of emission signal is $\mathrm{B}$, cycle is $\mathrm{T}$, the following relation is made:

$$
\frac{T}{B}=\frac{t_{d}}{f_{b}}=\frac{2 d}{c \cdot f_{b}} \Longrightarrow d=\frac{T \cdot c \cdot f_{b}}{2 B}
$$

c is the electromagnetic wave's velocity in air, usually considered to be equal to approximately speed of light. Then we can obtain target distance $d$ according to frequency of IF signal after mixing.

\section{Effects of VCO nonlinearity on distance resolution}

Calculating a derivative of Equation (1), we can deduce distance resolution equation of FMCW $\operatorname{radar}^{[7]}$

$$
\Delta d=\frac{T \cdot c}{2 B} \delta f_{b}
$$

$\delta f_{b}$ is bandwidth of beat IF signal. When sending ideal linear FM signal, beat signal is single frequency one in an effective cycle, its bandwidth $\delta f_{b}$ is about inverse of cycle T. Now the distance resolution from Equation (2) is 


$$
\Delta d=\frac{c}{2 B}
$$

$\Delta d$ is ultimate resolution of FMCW radar. Its meaning is, when emission signal is ideal linear FM signal, distance resolution is determined by bandwidth of emission signal.

In literature [7] and [8], when nonlinearity of FM source increases, distance resolution will reduce with it. Although nonlinearity is small, it can cause radar distance resolution decrease, even can't find targets ${ }^{[9]}$. FM linearity of radar is decided greatly by VCO nonlinearity degree. So high linearity of VCO is very important to the whole system performance.

In general conditions, VCO Tuning Characteristics are all nonlinear, even Tuning Characteristics are good, its nonlinearity also exceed 5\%. So we must correct VCO nonlinearity beforehand in linear FMCW radar system.

\section{Main work about the paper}

The main work about the paper is against forepart of automobile collision warning system which works near $60 \mathrm{~Hz}$. We have designed a simple, efficient method of VCO linearity correction and put it into hardware implementation.

\section{VCO LINEARITY CORRECTION METHOD}

\section{A. Open-loop nonlinearity correction principle}

Considering three common methods of VCO correction: reactance compensation linearity correction ${ }^{[10]}$, open loop linearity correction ${ }^{[11]}$ and closed loop linearity correction ${ }^{[12,13]}$, we chose the simplest and also the most pertinent open loop linearity correction. In relation to negative feedback of closed loop nonlinearity correction, open loop linearity correction use corrected VCO voltage control curve as entry control of VCO. And it makes output frequency and time appearing linear relationship in a FM cycle, that's ideal linearity FM.

We assume that Tuning Characteristic of VCO is

$$
f(\nu)=f_{0}+\mu \nu+e(\nu)
$$

$f_{0}$ is natural harmonic frequency when control voltage is 0 $\mu$ is average slope of Tuning Characteristic and it's a constant. The maximum value of input control voltage is assumed $v_{\max }$, the corresponding output maximum frequency is $f_{\max }$, so the definition of $\mu_{\text {is }}$

$$
\mu=f_{\max } / \nu_{\max }
$$

$e(v)$ is nonlinear term of tuning characteristic. When $e(v)$ is equal to 0 identically, VCO is ideal linearity FM and if nonlinearity is existing, $e(v)$ will not be equal to $0^{[14]}$.

Obviously, when input control voltage is linearly increasing relative to time $t$ in a cycle, $v_{c}(t)=a t$

$a$ is increasing rate of voltage and $a>0$ 。Inserting input control voltage of equation (6) in equation (4) and the relation of output frequency and time will be

$$
f(t)=f_{\mathrm{o}}+\mu \cdot a \cdot t+e(a t)=f_{\mathrm{o}}+\mathrm{A} t+e(a t)
$$

If $e(v) \neq 0, f(t)$ isn't linear FM. That means, when nonlinearity of VCO exists, use linear signal controlling VCO, then output signal is not linear FM. Because changing tuning characteristics is more difficult, our work is to find a input control voltage curve $v_{c}^{\prime}(t)=a t+\delta(t)$ and let VOC output linear FM signal under control of $v_{c}^{\prime}(t)$.

$$
f(t)=f\left(v_{c}^{\prime}(t)\right)=f_{0}+\mu \cdot a \cdot t+\mu \cdot \delta(t)+e(a t+\delta(t)) \equiv f_{0}+\mathrm{B} t
$$

The actual operation method is as figure 3 shown.
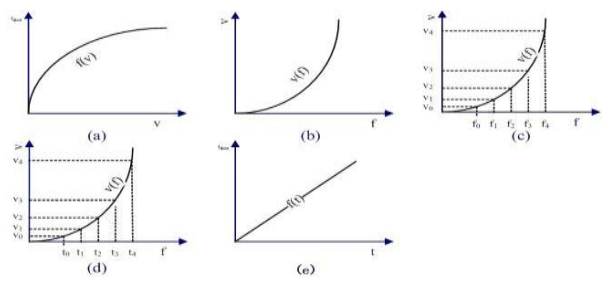

Figure 3. Diagram of open-loop nonlinearity correction

Our purpose is to acquire control voltage sequence $\left(v_{0}, v_{1}, v_{2}, \cdots v_{n-1}\right)$ used to correct VCO nonlinearity.

\section{B. Acquisition of VCO nonlinearity correction curves}

1) Measurement of VCO nonlinearity Tuning Characteristics curves

VCO nonlinearity Tuning Characteristics curves will be obtained through combination of measurement and numerical computation. As Figure 4(a) shown, we choose voltages as many as we can in reasonable scope. And use these voltages as control voltages of VCO in turn, measure corresponding VCO output frequency of every control voltage by spectrometer, finally form a series of data $\left(v_{i}, f_{i}\right)$. According to these data, we hope to construct VCO tuning characteristics within the range of control voltages.

Because our purpose of measuring tuning characteristics $f(v)$ is to get its inverse function $v(f)$, we might take $\mathrm{f}$ as variables to form new data and using $\left(f_{i}, v_{i}\right)$ to fit $v(f)$, as Figure 4 shown.

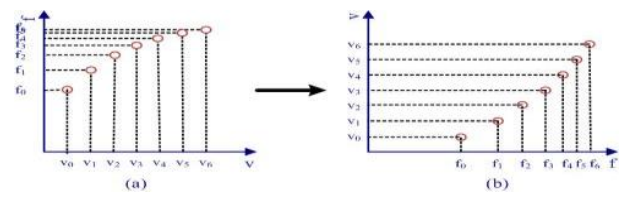

Figure 4. Data measurement and conversion of VCO Tuning Characteristics 
Figure 5 is the diagram of using $\left(f_{i}, v_{i}\right)$ to fit $v(f)$. In order to achieve this goal, we need to calculate with help of interpolation.

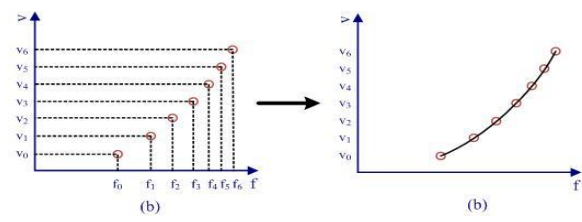

Figure 5. Fit Function Curve using discrete dada points

\section{2) Acquisition of VCO nonlinearity correction data}

After we acquired a whole curve $f(v)$ with method of linear interpolation, we evenly chose $\mathrm{M}$ points in output frequency interval.

In practice, we use interpolation function Interp1 of Matlab 7.4 to calculate linear interpolation. Call form of Interp1 is:

$$
\mathrm{YI}=\operatorname{INTERP1}(\mathrm{X}, \mathrm{Y}, \mathrm{XI}, \mathrm{METHOD})
$$

$\mathrm{X}, \mathrm{Y}$ is respectively abscissa vector and ordinate vector through measurement.

$$
X=\left(f_{0}, f_{1}, f_{2}, \cdots f_{N-1}\right), Y=\left(v_{0}, v_{1}, v_{2}, \cdots v_{N-1}\right)
$$

$\mathrm{N}$ is numbers of measured data points.

$\mathrm{XI}$ is abscissas of various points, METHOD is method of interpolation and YI is corresponding ordinate vectors by calculation.

What we want is corresponding control voltage values when VCO output frequency evenly increase, that's samples of $v(f)$.As shown in Figure 6.
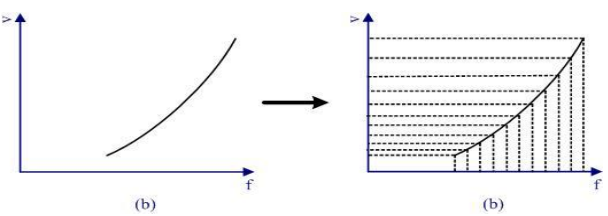

Figure 6. Sample of $v(f)$ curve XI:

When number of sampling points is $\mathrm{M}$, the expression is of

$$
X I=f_{\min }:\left(f_{\max }-f_{\min }\right) /(M-1): f_{\max }
$$

Make use of data points from measurement and XI; we can obtain control voltage sequence by introducing Interp1 function.

\section{Design of hardware solution}

After we got voltage sequence, the goal of hardware solution need to achieve is, under clock control of fixed frequency, use these voltages as VCO input control voltages in turn in a circle. In consideration of the actual conditions, the block diagram of hardware solution we designed is as shown in Figure 7.

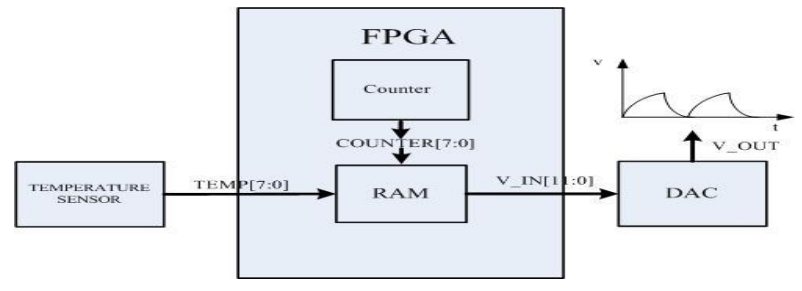

Figure 7. Functional block diagram of hardware solution

The analog signals used to correct VCO will be changed to 12 bit digital signals at first and stored in RAM. Under control of clock, counter begin to count and output 8 bit counting value COUNTER. At the same time, the temperature transducer output 8 bit signal which can reflect current temperature in real time. These 16 bit signals decide 12 bit numerical value input into digital-to-analog converter DAC. DAC transfer digital signals V_IN into analog ones V_OUT in real time, as input voltages to control $\mathrm{VCO}$ and correct nonlinearity.

Figure 7 is just a block diagram, practical hardware implementation need more other elements. For example,

a)

$60 \mathrm{GHz}$ VCO : VCO is core element of linear FM radar system. The VCO we used is millimeter wave varactor produced by Quinstar Technology; its model is DTVOG170V. Under control voltage from $0 \mathrm{~V}$ to $15 \mathrm{~V}$, the sweptfrequency bandwidth is about $700 \mathrm{MHz}$ and its range is $59.536-$ $60.266 \mathrm{GHz}$.

b) DS18B20 temperature transducer: We used is DS18B20 produced by Dallas Semiconductor. At the temperatures ranging from $-55^{\circ}$ to $+125^{\circ}$, it can provide $9-12$ bit digital output to indicate current temperature. The conversion cycle is less than $750 \mathrm{~ms}$. Its real-time performance is good.

c) AD5445 digital-to-analog converter: DAC we used in this subject is AD5445 made by Analog Device. AD5445 is a digital-to-analog converter of 12 bit digital input and output current based on CMOS Technology.

d) Spectrum analyzer: We used to observe VCO output frequency is $8563 \mathrm{EC}$ portable color microwave spectrum analyzer made by Agilent. This analyzer has standard frequency range from $9 \mathrm{kHz}$ to $26.5 \mathrm{GHz}$ and covered by optional low end frequency to $30 \mathrm{~Hz}$. When using heterodyne mixing, the frequency range is from $18 \mathrm{GHz}$ to $325 \mathrm{GHz}$.

\section{MODULE DESIGN BASED ON FPGA}

\section{A. Temperature transducer driver module}

The temperature transducer driver module is to realize communication between FPGA and DS18B20 chip. FPGA gave an instruction and asked DS18B20 to measure temperature. After answered and measured, DS18B20 sent the result back to FPGA. Because DS18B20 and FPGA connected by only one dateline, data need to be transmit in bit. So communication process must strictly obeyed communication protocol. 
According to operation sequence and procedure of DS18B20, we designed a flow sheet which can complete a temperature conversion and read temperature output. This procedure could be realized by a finite state machine. The state transition diagram is as Figure 8 illustrated.

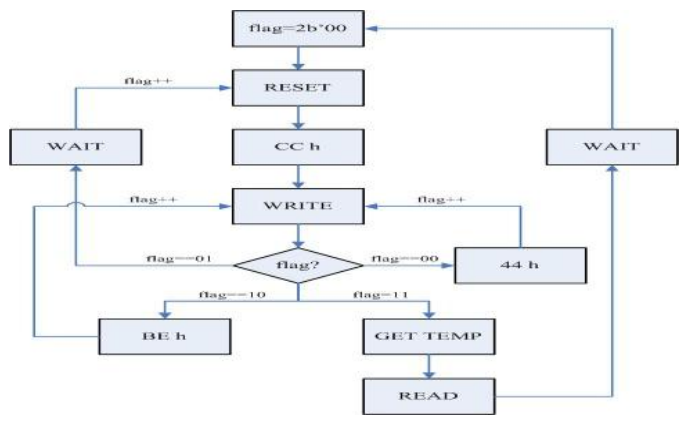

Figure 8. Status transfer diagram of DS18B20 finite state machine

\section{B. VCO tuning characteristics curve measurement module}

In order to get VCO tuning characteristics curve, we need to use ladder-type voltage as VCO input control voltage and record output frequency under every voltage. But in the actual operating process, 12 bit digital signal saved in FPGA as DAC input decides VCO control voltage every moment. We might directly output digital signals which are increasing evenly through FPGA as DAC input and measure corresponding VCO input frequency.

Because as DAC input, the min value of digital signals is 0 of 12 bit, max value is 1 of 12 bit, that's 212-1. We choose 64 as interval and then could collect 65 points: $0,64,128, \ldots, 212-$ 64,212-1. Beforehand we store these points in RAM, then using FPGA output these numbers to DAC in order and create corresponding analog control voltages.

In order to increase operability and measure output frequency conveniently, we add a controller for data-chosen. Every time when data-chosen changed from high-level voltage to low-level voltage, output increased 64 until figures become 212-1, although outputs remain unchanged. And we also add a catch module to catch the change of data-chosen.

\section{VCO nonlinearity correction module}

The function of VCO nonlinearity correction module is to let FPGA output digital correction burst, and use these data as DAC input to generate analog voltage signals and control VCO output frequency.

Nonlinearity correction module and measurement module are relatively similar. But there are also some differences. At first, in measurement module, output data are changed manually and in correction module, correction data output automatically and periodically. Secondly, temperature transducer module is only used to observe temperature, but in correction module temperature transducer decide FPGA current output with clock.

Nonlinearity correction module ad5445Tri could make DAC output approximate triangular-wave and according to change of temperature choose proper data set. We supposed that under a certain temperature, we obtain correction data sequence $\left(a_{0}, a_{1}, a_{2}, \cdots, a_{M-1}\right)$ using interpolation and so FPGA may output digital signal: $a_{0}, a_{1}, a_{2}, \cdots, a_{M-2}, a_{M-1}, a_{M-2}, \cdots, a_{2}, a_{1}, a_{0}$ to $\mathrm{DAC}$ periodically.

In fact, because of discrete correction data, so frequency change is also discrete. FM signals output by VCO is approximate sawtooth triangular-wave like Figure 9 shown. Adding sample points of correction data could make signals become smooth.

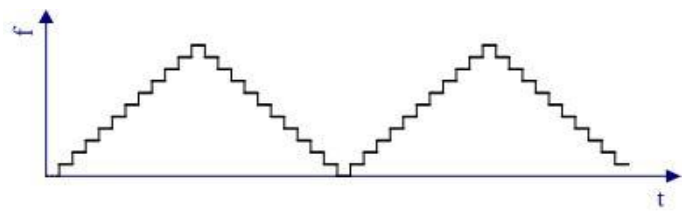

Figure 9. FM signal output actually by VCO

\section{SUMMARY}

\section{A. Measurement of VCO tuning characteristics}

The circuit diagram is shown in Figure 10. VCO, DAC and FPGA all use the voltage stabilizing circuit stated in CHAPTER II as supply power and input reference voltage of DAC is $10 \mathrm{~V}$. Chip DS18B20 attached to VCO surface by insulating tape to measure temperature of transducer and output the result displayed by seven-segment digital tube. The output of VCO is coupled to input port of spectrum analyzer through attenuator and waveguide.

After correction by spectrum analyzer, till VCO realize thermal balance, if there's no frequency spectrum drift occurs and begin to measure.

We could change digital input of FPGA manually using data-chosen-switch and record output peak frequency of VCO. In Figure 11, ordinate is FPGA output and abscissa is VCO output frequency.

We use Matlab curve fitting toolbox for linear interpolation fitting the result is as Figure 12 shown. It can be seen that large portion of curve has good linearity and some points behind part occur significant nonlinearity. When acquiring correction data, we should exclude these points; only choose the curve with good linearity and the frequency range is from 59.62314 to $59.95977 \mathrm{GHz}$.

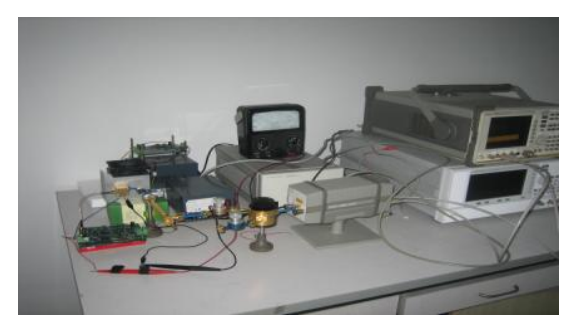

Figure 10. Electric circuits of experiments 


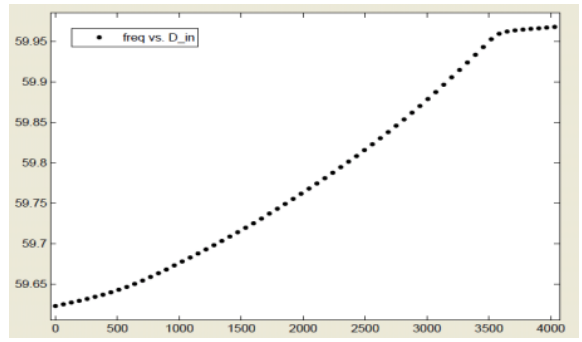

Figure 11. VCO tuning characteristics measurement value

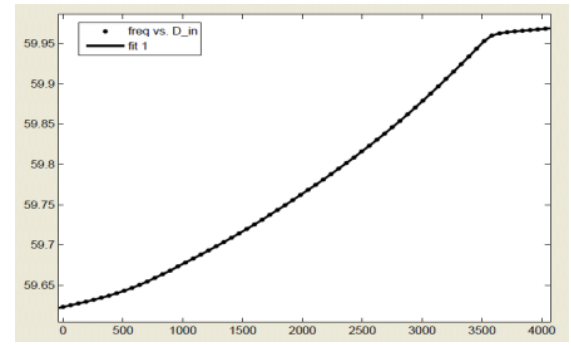

Figure 12. VCO tuning characteristics curve after linear interpolation fitting

If taking the corresponding linearity input voltage of these measured points as VCO input control, under clock frequency of $1 \mathrm{KHz}$. The contrast diagram of VCO FM curve and ideal FM curve is as shown in Figure 13. The average frequency error in half a circle is $32.6 \mathrm{MHz}$, comparing with $336.6 \mathrm{MHz}$ bandwidth, the average nonlinearity is $9.7 \%$. So there exists quite apparent nonlinearity.

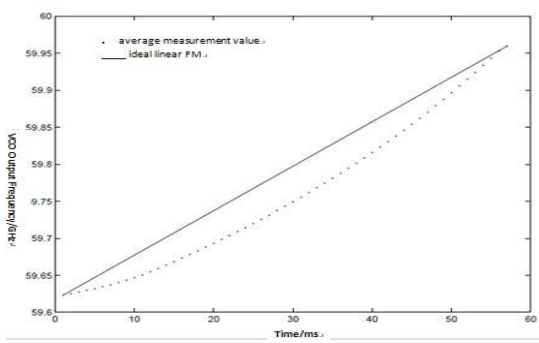

Figure 13. Contrast of VCO FM curve and ideal linear FM curve

\section{B. VCO nonlinearity correction}

We extract 65 frequency points from range of 59.62314 to $59.95977 \mathrm{GHz}$ using method mentioned in CHAPTER II and obtain values of digital signals as correction data for VCO nonlinearity. Then store these data in ROM and call VCO nonlinearity correction module to correct $\mathrm{VCO}$ output frequency periodically.

But VCO instantaneous output frequency is unmeasurable; for verifying correctness of correction scheme, we still need to output correction data manually using data-chosen-switch in order according to method of VCO tuning characteristics measurement. To validate correction stability, we measured twice under the same temperature and contrast of average value of results and ideal linearity FM curve is shown in Figure 14

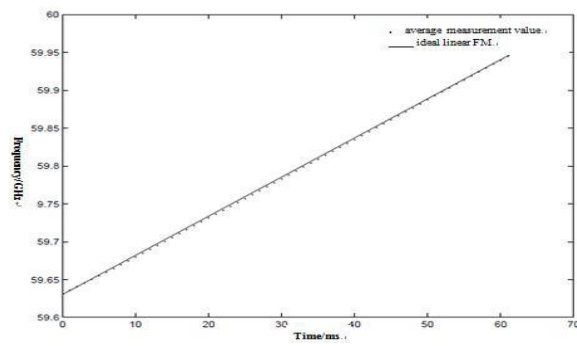

Figure 14. Measurement data after nonlinearity correction and ideal linear FM curve

Comparing with ideal FM, the maximum frequency difference of $\mathrm{VCO}$ are less than $3 \mathrm{MHz}$ at any time after linearity correction. Relative to $320.7 \mathrm{MHz}$ bandwidth near $60 \mathrm{GHz}$, the average error is $1.9 \mathrm{MHz}$ and average nonlinearity is only $0.6 \%$.

VCO nonlinearity correction system designed in this project has good performance and the VCO nonlinearity could descend more than an order of magnitude. Use of temperature transducer can improve adapting ability of this system at different room temperature and reduce correction error.

[1] Liu Guosui, Sun Guangmin, Gu Hong, Zhu Xiaohua, CW radar and its signal processing technique, Fire Control Radar Technology, 1995, $24: 20-31$

[2] Zheng Wei, Lu Guanghua, Chen Weidong, Liu Falin, Novel Method for Linearity Correcting and System Application, Fire Control Radar Technology, 2005, $34: 12-15$

[3] Yu Pei, Millimeter Wave linear FM radar system analysis and VCO design, 2006

[4] SMULDERS P, Exploiting the $60 \mathrm{GHz}$ Band for Local Wireless Multimedia Access: Prospects and Future Directions[J], IEEE Communications Magazine, 2002, 40(1): 140-147.

[5] OHATA K., MARUHASHI K., ITO M., et al., $1.25 \mathrm{Gbps}$ Wireless Gigabit Ethernet Link at 60GHz-Band, 2003 IEEE MTT-S International Microwave Symposium Digest, 2003: 372376.

[6] Huang Tao, Journal of Detection \& Control, 2002, 24(3) : 2932.

[7] Wang Zonghuan, Key technique of continuous wave radar, LIBAYI KEJI, 2002, 2 : 20-28.

[8] Song Jingwei, Linearity Effects on Linear Radar Distance Resolution, Journal of University of Electronic Science and Technology of China, 1992, 21(2),121-136.

[9] Wang Xuegang, Feng Jianxiong, Xiang Jingcheng, Linearity Correction For Linear FM Sweep Signals, ACTA ELECTRONIC SINICA, 1996, 24(10), 120-122.

[10] Lockyear W H, Linearize VCOs by Reactance Compensation, Microwave J, 1980, 23 (2): 60-68.

[11] Buswell R N, Behind the design of VCO linearizers, Microwave, 1976, 15(9): 36-40.

[12] Blazer R J, Analog multipliers drive component linearization scheme, Microwave\&RF, 1992, 31(10):74-80.

[13] Watkins Johnson Co, Digitally tuned PROM-corrected VCO, Microwave J, 1982, 25(11): 131-132.

[14] Huang Renxin, Zong Chengge, Research on a kind of VCO nonlinearity compensation method, Control and Instruments In Chemical Industry, 2004, 31(3) : 50-51. 